\title{
Alimentos transgênicos: conhecimento entre acadêmicos de nutrição
}

Food transgenic: knowledge between students of nutrition

Marislane Campos da Silva ${ }^{1}$, Carolina Abreu de Carvalho², Ângela Tamara Souza Barroqueiro $^{2}$, Rosangela Maria Lopes de Sousa ${ }^{2}$, Ana Carolina Pereira de Castro ${ }^{3}$, Alexsandro Ferreira dos Santos ${ }^{4}$.

Resumo: O surgimento de Organismo Geneticamente Modificados na produção de alimentos tem estado em evidência atualmente, causando polêmicas e gerando debates mundiais. A falta de informações precisas, pode estar relacionada com a falta de problematização no espaço acadêmico. Objetivo: Descrever a percepção de sobre alimentos transgênicos entre alunos do primeiro e do último semestre do curso de Nutrição de uma Universidade particular de São Luís, Maranhão. Materiais e métodos: Estudo transversal e descritivo, realizado com 72 alunos do primeiro e último semestre do curso de Nutrição de uma Universidade particular de São Luís, Maranhão, com matricula ativa em maio de 2016. Para a coleta de dados, utilizou-se um questionário adaptado de acordo com os objetivos da pesquisa. Os dados foram apresentados na forma de tabelas, em frequências absolutas e relativas. Resultados: $\mathrm{A}$ análise dos dados, mostrou que: $87,5 \%$ dos avaliados eram mulheres, $84,7 \%$ apresentaram conhecimento sobre o conceito de alimentos transgênicos, quanto ao meio de divulgação, a internet foi citada por $68,1 \%$ dos acadêmicos. Verificou-se que mais de um terço dos alunos não conhecia a simbologia de alimentos transgênicos. Ao questionar sobre 0 interesse pelo tema, destacou-se uma maior prevalência (58,3\%) para o interesse moderado, $85,7 \%$ dos avaliados no primeiro semestre não foram favoráveis ao consumo e $55,6 \%$ no último período foram favoráveis. Conclusão: Houve elevado conhecimento sobre alimentos transgênicos, no entanto esse conhecimento parece ser superficial e não definido.

Palavras-chave: Alimentos transgênicos. Nutrição. Conhecimento.

\begin{abstract}
The emergence of genetically modified organism in food production has been in evidence today, causing controversy and creating global debates. The lack of accurate information may be related to the lack of questioning the academic space. Objective: To describe the perception of nutrition on transgenic foods among students of the first and last half of the Nutrition course of a particular University of São Luís, Maranhao. Materials and methods: cross-sectional descriptive study conducted with 72 students of the first and last half of the Nutrition course in a private university of São Luís- Ma, with active registration in May 2016. To collect data we used an adapted questionnaire according to the research objectives. The data were presented in tables, in absolute and relative frequency. Results: The results showed that $87.5 \%$ of the individuals were female, had knowledge of the concept of transgenic food (84.7\%), as the means of dissemination, the Internet was cited by $68.1 \%$ of the students. It was found that more than a third of the students did not know the symbolism of transgenic foods. When questioned about the interest in the subject, the highlight was a higher prevalence $(58.3 \%)$ to moderate interest $(85.7 \%)$ of the evaluated in the first half were not favorable to consumption and $55.6 \%$ in the last period were. Conclusion: There was a high knowledge about transgenic foods, however this knowledge is superficial and not set.
\end{abstract}

Keywords: Transgenic food. Nutrition. Knowledge

1 - Acadêmica do Curso de Nutrição - Universidade Ceuma

2,3 e 4 - Docentes do Curso de Nutrição - Universidade Ceuma

Rev. Investig. Bioméd. São Luís, 9:31-39, 2017 
Introdução

Os alimentos transgênicos podem ser definidos como organismos vivos, cujo material genético foi modificado artificialmente. $O$ processo ocorre por meio da transferência de genes selecionados, individualmente, de um organismo ao outro, ou entre espécies distintas, utilizando-se assim da engenharia genética. $O$ surgimento de Organismos Geneticamente Modificados (OGMs) na produção de alimentos tem estado em evidência atualmente, causando polêmicas e gerando debates mundiais ${ }^{1}$.

De acordo com o Instituto de Defesa do Consumidor, o Brasil tem a segunda maior produção de transgênicos do mundo. Segundo o órgão, em 2012, cerca de $50 \%$ do algodão, $76 \%$ do milho e $89 \%$ da soja cultivados no país eram geneticamente modificados ${ }^{2}$.

É inegável que a evolução da ciência é dinâmica, e que os OGMs já estão no mercado em grande escala $^{3}$. Tal realidade aponta uma escassez significativa de pesquisas sobre os transgênicos. A falta de informações precisas, podem estar relacionadas com a ausência de problematização no espaço acadêmico sobre as inovações tecnológicas que envolvem riscos, 0 que consequentemente, leva à maior carência de dados para 0 consumidor final ${ }^{4}$.

Diante da deficiência de informação da população que vem se destacando em estudos ${ }^{5,6}$. É indispensável avaliar o nível de conhecimento existente pelos alunos de graduação em Nutrição sobre os alimentos transgênicos, por ser de grande utilidade em termos de conscientização destes futuros profissionais. Podendo ainda, contribuir e influenciar na melhoria da grade curricular dos mesmos, instigando-os ao aprofundamento dos estudos em torno da temática, 0 que proporcionará, maiores esclarecimentos ao público consumidor. Visto que 0 conhecimento que se tem sobre a problemática dos transgênicos ainda é reduzido.

Assim 0 presente estudo objetivou descrever a percepção de nutrição sobre alimentos transgênicos entre alunos do primeiro e do último semestre do curso de Nutrição de uma Universidade particular de São Luís, Maranhão.

\section{Materiais e Métodos}

Realizou-se um estudo transversal descritivo com 72 alunos do curso de Nutrição.

O período de coleta aconteceu no mês de maio de 2016, nos turnos matutino e vespertino, do curso de Nutrição da Universidade Ceuma, Campus Renascença, em São Luís, Maranhão, Brasil.

O público alvo da pesquisa foram os acadêmicos do primeiro e do último semestre do curso de Nutrição com matrícula ativa na Universidade Ceuma.

A coleta de dados foi realizada através da aplicação de um questionário, adaptado de Quesada e Lembo $^{3}$ de acordo com os objetivos da pesquisa, contendo informações sobre os avaliados (sexo, idade e período) e questões de múltipla escolha, onde abordou 0 
interesse e o conhecimento sobre alimentos transgênicos.

Os dados foram coletados por amostra não probabilística. Iniciouse a coleta logo após autorização do local de pesquisa e o convite da pesquisadora aos alunos em sala a participar da pesquisa, sem prejuízo em suas atividades acadêmicas, em horário extra. Neste momento, os avaliados foram informados dos benefícios e da importância em colaborar com o presente estudo. Os alunos que aceitaram participar da pesquisa, assinando o Termo de Consentimento Livre e Esclarecido, responderam individualmente as perguntas, em um período máximo de 15 minutos.

Os dados coletados foram registrados e tabulados no Microsoft Office Excel® 2013, e logo após, analisados no programa estatístico Bioestat ${ }^{\circledR} \quad$ versão 5.3 da Universidade Federal do Pará.

Os resultados foram apresentados na forma de tabelas, em frequências absolutas e relativas.

O estudo respeitou as diretrizes e critérios estabelecidos na Resolução 466/12 do Conselho Nacional de Saúde ${ }^{7}$, nos preceitos éticos estabelecidos da pesquisa com seres humanos, tendo aprovação do Comitê de Ética da Universidade Ceuma sob o número de protocolo no 1.516.254.

\section{Resultados}

Entre os 72 acadêmicos avaliados, $87,5 \%$ eram do primeiro semestre e $12,5 \%$ do último, notouse maior frequência de mulheres (acima de $90 \%$ para ambos os semestres). A faixa etária prevaleceu entre 16 a 25 anos $(85,7 \%)$ no primeiro semestre e $55,6 \%$ para o último semestre de graduação (Tabela 1).

Tabela 1 - Características de estudantes de uma Universidade particular. São Luís, Maranhão, 2016.

\begin{tabular}{lccc}
\hline \multirow{2}{*}{ Variáveis } & \multicolumn{2}{c}{ Semestre de graduação } & Total \\
\cline { 2 - 3 } & $\begin{array}{c}\mathbf{1}^{\mathbf{0}} \text { período } \\
\mathbf{n}(\%)\end{array}$ & $\begin{array}{c}\mathbf{8}^{\circ} \text { período } \\
\mathbf{n}(\%)\end{array}$ & $\mathbf{n}(\%)$ \\
\hline Sexo & $6(9,5)$ & $0(0,0)$ & $6(8,3)$ \\
Masculino & $57(90,5)$ & $9(100,0)$ & $66(91,7)$ \\
Idade & $54(85,7)$ & $5(55,6)$ & $59(81,9)$ \\
16 a 25 anos & $7(11,1)$ & $1(11,1)$ & $8(11,1)$ \\
26 a 35 anos & $2(3,2)$ & $3(33,3)$ & $5(7,0)$ \\
$>35$ anos & $63(87,5)$ & $9(12,5)$ & $72(100,0)$ \\
\hline Total & &
\end{tabular}


Ocorreu maior frequência $(84,7 \%)$ de conhecimento sobre o conceito de alimentos transgênicos. Entre os alunos do primeiro semestre $33,3 \%$ estudaram o tema no ano corrente, enquanto $66,7 \%$ dos alunos do último semestre, não lembra quando estudaram 0 assunto. Dentre os avaliados do primeiro semestre, ao menos metade $(52,4 \%)$, apresentaram um grau de conhecimento médio sobre o assunto. No que tange ao meio de divulgação, a internet foi citada por $68,1 \%$ dos alunos. Verificou-se que mais de um terço dos avaliados não conhecia a simbologia aplicada na rotulagem de alimentos transgênicos, tanto no primeiro semestre quanto no último semestre de graduação (Tabela 2).

Tabela 2 - Avaliação do conhecimento sobre alimentos transgênicos em estudantes do primeiro e último semestre de graduação em Nutrição. São Luís, Maranhão, 2016.

\section{Variáveis}

\begin{tabular}{|c|c|c|}
\hline período & 8 período & Total \\
\hline
\end{tabular}

\section{Grau de interesse}

Sem interesse

Interesse moderado

Interesse extremo

$\begin{array}{lrlllrl}\text { Sem interesse } & 1 & (1,6) & 0 & (0,0) & 1 & (1,4) \\ \text { Interesse moderado } & 35 & (55,6) & 7 & (77,8) & 42 & (58,3) \\ \text { Interesse extremo } & 27 & (42,8) & 2 & (22,2) & 29 & (40,3) \\ \text { avorável ao consumo } & & & & & & \\ \text { Sim } & 9 & (14,3) & 5 & (55,6) & 14 & (19,4) \\ \text { Não } & 54 & (85,7) & 4 & (44,4) & 58 & (80,6)\end{array}$

Favorável ao consumo

Preocupação com os

efeitos

$\begin{array}{lclllrl}\text { Sim } & 62 & (98,4) & 9 & (100,0) & 71 & (98,6) \\ \text { Não } & 1 & (1,6) & 0 & (0,0) & 1 & (1,4)\end{array}$

Auxílio dos transgênicos para a população

\section{humana}

Sim

Não

Sem opção

$11 \quad(17,5) \quad 1 \quad(11,1) \quad 12 \quad(16,7)$

$\begin{array}{llllll}41 & (65,0) \quad 5 & (55,6) & 46 & (63,9)\end{array}$

$\begin{array}{llllll} & (17,5) & 3 & (33,3) & 14 & (19,4)\end{array}$

Concordância com o

consumo sem

conhecimento prévio

\begin{tabular}{ccccccc} 
Sim & 62 & $(98,4)$ & 9 & $(100,0)$ & 71 & $(98,6)$ \\
Não & 1 & $(1,6)$ & 0 & $(0,0)$ & 1 & $(1,4)$ \\
\hline Total & $63(87,5)$ & $9(12,5)$ & $72(100,0)$ \\
\hline
\end{tabular}


Sobre o interesse pelo tema observou-se maior prevalência $(58,3 \%)$ para o interesse moderado. Ao avaliar se os acadêmicos eram favoráveis ao consumo de alimentos transgênicos, notou-se que no primeiro semestre, $85,7 \%$ dos avaliados não foram favoráveis ao consumo de alimentos transgênicos, no último semestre $55,6 \%$ foram a favor. A maioria dos avaliados $\quad(98,5 \%) \quad$ tinham preocupação com os efeitos de alimentos transgênicos no organismo humano. Sobre o auxílio dos transgênicos para a população, destacou-se $63,9 \%$ para a resposta negativa. Em relação a concordância com o consumo sem conhecimento prévio, 98,6\% dos avaliados manifestaram-se positivamente (Tabela 3).

Tabela 3 - Avaliação do interesse sobre alimentos transgênicos por estudantes de Nutrição. São Luís, Maranhão, 2016.

\begin{tabular}{|c|c|c|c|}
\hline Variáveis & $\begin{array}{c}\text { 1\% período } \\
\text { n (\%) }\end{array}$ & $\begin{array}{c}8^{\circ} \text { período } \\
\text { n (\%) }\end{array}$ & $\begin{array}{l}\text { Total } \\
\text { n (\%) }\end{array}$ \\
\hline \multicolumn{4}{|c|}{ Conhecimento do conceito } \\
\hline Sim & $53(84,1)$ & $8(88,9)$ & $61(84,7)$ \\
\hline Não & $10(15,9)$ & $1(11,1)$ & $11(15,3)$ \\
\hline \multicolumn{4}{|l|}{ Período de estudo } \\
\hline No ano em curso & $21(33,3)$ & $0(0,0)$ & $21(29,2)$ \\
\hline Não lembra & $19(30,2)$ & $6(66,7)$ & $25(34,7)$ \\
\hline Ano passado & $15(23,8)$ & $1(11,1)$ & $16(22,2)$ \\
\hline Há muito tempo & $8(12,7)$ & $2(22.2)$ & $10(13,9)$ \\
\hline \multicolumn{4}{|l|}{ Grau de conhecimento } \\
\hline Muito & $3(4,7)$ & $0(0,0)$ & $3(4,2)$ \\
\hline Pouco & $25(39,7)$ & $4(44,4)$ & $29(40,3)$ \\
\hline $\begin{array}{l}\text { Vledio } \\
\text { Nada }\end{array}$ & $33(52,4)$ & $3(33,3)$ & $36(50,0)$ \\
\hline $\begin{array}{l}\text { Nada } \\
\text { Meio de divulqacão }\end{array}$ & $2(3,2)$ & $2(22,2)$ & $4(5,5)$ \\
\hline consultado & & & \\
\hline Livro didático & $6(9,5)$ & $2(22,2)$ & $8(11,1)$ \\
\hline Internet & $43(68,3)$ & $6(66,7)$ & $49(68,1)$ \\
\hline TV & $6(9,5)$ & $0(0,0)$ & $6(8,3)$ \\
\hline Revistas & $3(4,7)$ & $0(0,0)$ & $3(4,2)$ \\
\hline $\begin{array}{l}\text { Revistas } \\
\text { Outros (artigos, etc.) }\end{array}$ & $5(8,0)$ & $1(11,1)$ & $6(8,3)$ \\
\hline \multicolumn{4}{|c|}{ Conhecimento do símbolo } \\
\hline Sim & $39(61,9)$ & $6(66,7)$ & $45(62,5)$ \\
\hline Não & $24(38,1)$ & $3(33,3)$ & $27(37,5)$ \\
\hline Total & $63(87,5)$ & $9(12,5)$ & $72(100,0)$ \\
\hline
\end{tabular}




\section{Discussão}

Dentre os participantes da pesquisa verificou-se maior prevalência de mulheres, com faixa etária entre 16 a 25 anos nos dois grupos estudados. Constatou-se também elevada frequência de indivíduos que conhecem o conceito de alimentos transgênicos, esses dados são semelhantes ao de Oliveira e Nojimoto ${ }^{1}$, em seu estudo realizado com alunos e frequentadores de dois supermercados de Goiânia, que avaliou o perfil de conhecimento sobre alimentos transgênicos e observou que $91 \%$ dos alunos demonstram conhecimento superior sobre esses alimentos. Considera-se que tais resultados são oriundos do grau de escolaridade dos entrevistados.

Ao questionar o momento em que estudaram alimentos transgênicos, notou-se em boa parte dos estudantes, o relato de "não se lembrar" caso tenha estudado ou feito leitura sobre o assunto. Percebeu-se que, mesmo na atualidade com repletas descobertas científicas e tecnológicas, polemizado no âmbito escolar e divulgadas no meio acadêmico de forma a fazerem parte do cotidiano, a amostra ainda se encontra insegura para emitir opiniões fundamentadas ${ }^{8}$.

No que diz respeito ao conhecimento sobre os transgênicos, poucos dos pesquisados lembram-se muito dos conceitos já estudados, achados estes condizentes com 0 estudo de Quesada e Lembo ${ }^{3}$ no qual foi observado que somente $9 \%$ dos estudantes da área da saúde lembravam o que aprenderam. Esses percentuais podem significar deficiência no aprendizado, ou ainda a ausência de estudos mais elaborados a cerca do tema. A internet foi a principal mídia responsável pela divulgação de informações aos interessados sobre OGMs. Acredita-se que a mídia, referida como fonte de origem das informações, não tem enfocado o tema profundamente, acompanhando a polêmica de forma bastante restrita, muitas vezes desprovida de saber científico ${ }^{9}$.

Ao indagar sobre a identificação da simbologia de transgênicos, o estudo apontou maior prevalência de acadêmicos que sabiam identificar, no entanto não se esperava que $37,5 \%$ dos alunos desconhecessem 0 símbolo, visto que fazem parte de um grupo mais seleto, com acesso a informações mais refinadas. Farias e colaboradores $^{5}$ notaram em seu estudo com 45 estudantes de uma universidade pública no Rio de Janeiro (Brasil), que $20 \%$ dos estudantes de graduação da Universidade do Estado do Rio de Janeiro responderam que conseguiriam reconhecer alimentos transgênicos a partir da embalagem do alimento.

Referente ao interesse pelo tema, quase totalidade da amostra demonstraram ter entusiasmo frente ao assunto, resultado que corroborou com o estudo de Quesada e Lembo ${ }^{3}$ onde percebeu-se existir amplo interesse sobre o tema. O que diferem dos resultados encontrados por Scare e colaboradores ${ }^{10}$, em pesquisa com consumidores brasileiros, em que envolveu alunos e professores de cursos de graduação, observou-se que menos de $20 \%$ dos entrevistados se envolveriam mais com o assunto.

Quando os avaliados foram investigados quanto a concordância do consumo de alimentos transgênicos, houve uma certa indecisão em maior proporção no primeiro semestre, mas de maneira geral especificamente no último semestre os alunos se colocaram contra o consumo desses alimentos.

1 - Acadêmica do Curso de Nutrição - Universidade Ceuma

2,3 e 4 - Docentes do Curso de Nutrição - Universidade Ceuma 
Comumente são favoráveis ao consumo, aqueles que acreditam que esses alimentos não estão sujeitos a causar riscos à saúde, e por acreditar que poderiam combater a fome, em função do aumento de sua produção e por serem mais baratos. Aponta-se, entretanto, que 0 aumento da produção de alimentos não possibilitará a segurança alimentar e nutricional, uma vez que tal problema não decorre da produção, mas sim de sua distribuição para a população ${ }^{11}$.

Para aqueles que não são favoráveis ao consumo, a principal razão consiste no fato de não existir estudos científicos a longo prazo para esclarecer que benefícios e malefícios que 0 consumo desses alimentos possam trazer aos indivíduos.

Uma quantidade relevante de acadêmicos manifestou-se preocupados com os efeitos que os alimentos transgênicos possam causar no organismo humano, resultado semelhante foi encontrado no estudo de Quesada e Lembo ${ }^{3}$ em que 90\% dos alunos disseram se preocupar com os efeitos desses alimentos.

Considera-se esses dados decorrente da incerteza dos efeitos em longo prazo da ingestão de OGMs, e ainda insegurança, resultante da carência de debate aberto e vasto à sociedade sobre os possíveis riscos dessa inovação tecnológica, o que poderia acarretar na sua rejeição ${ }^{12}$.

A maioria dos avaliados na pesquisa acredita que a população brasileira consome alimentos geneticamente modificados sem prévio conhecimento. Arnaiz ${ }^{13}$, ao analisar pesquisas de opinião pública a respeito dos transgênicos, afirmou que, ainda, conhecemos pouco sobre o que comemos, e que as dúvidas da população a respeito dos OGMs provêm do comportamento das instituições responsáveis pela investigação dos possíveis riscos desses alimentos.

Neste contexto, evidenciamos a importância em aperfeiçoar o conhecimento dos integrantes do curso de Nutrição sobre o tema. Pois, este é um assunto cada vez mais presente no nosso cotidiano, mesmo parecendo ser desconhecido por futuros profissionais da área.

Atenta-se, que estes futuros profissionais, são responsáveis por manter a população atualizada sobre as inovações tecnológicas que envolvam alimentos geneticamente modificados.

Acredita-se que, as deficiências apontadas neste saber, possam ser solucionadas através de implementação de constante revisão pelos docentes nas disciplinas que acompanham tal conteúdo, sendo a grade curricular do curso, uma maneira de ajustar nas disciplinas, apontamentos mais precisos sobre 0 assunto.

Notou-se ainda, que são escassos os estudos que avaliam o conhecimento sobre alimentos transgênicos no grupo específico de graduação em Nutrição.

\section{Conclusão}

Houve elevado conhecimento sobre alimentos transgênicos, no entanto esse conhecimento é superficial e não definido. $O$ que chama atenção para a importância de maiores debates e explanação sobre o assunto no âmbito acadêmico, para que estes alunos, possam se posicionar criticamente sustentando uma posição ética em todas suas atividades.

As modificações na grade curricular buscando inserir disciplinas específicas que abordem 0 tema podem influenciar significativamente 
no aprendizado. $O$ estudo mostrou-se relevante e deve promover a realização de novas pesquisas em outras instituições de ensino superior para verificar a habilitação sobre este conteúdo no repasse de informações fundamentadas para o público.

Recomenda-se, que outros estudos sejam feitos para investigar se a ausência de disciplinas afinadas com o tema possa interferir no grau de conhecimento dos acadêmicos.

\section{Conflito de Interesse:}

Não houve conflito de interesses durante a realização do estudo.

\section{Referências}

1. Oliveira DM, Nojimoto, ITI. Perfil dos consumidores de alimentos transgênicos em Goiânia, Goiás. Revista de Nutrição e Vigilância em Saúde, Fortaleza, v. 1, n. 2, p. 26-29, 2014.

2. IDEC Instituto de Defesa do Consumidor. Festa Junina Transgênica. Testes e Pesquisas, v. 17, jun. 2013. Disponível em: <http://www.idec.org.br/consultas/t estes-e pesquisas/ festa-juninatransgenica>. Acesso em: 7 abr. 2016.

3. Quesada JCB, Lembo T. Percepção dos estudantes da área da saúde sobre biotecnologia e alimentos transgênicos. Journal of the Health Sciences Institute, São Paulo, v. 32, n. 3, p. 229-234, 2014.

4. Ribeiro IG, Marin VA. A falta de informação sobre os Organismos Geneticamente Modificados no Brasil. Ciência \& Saúde Coletiva,
Rio de Janeiro, v. 17, n. 2, p.359368, 2010.

5. Farias SCG, Filho, ST, Ribeiro, CRR, Araújo, MP, Viana, VJ, Farias, OLM. Percepção dos Alunos da Universidade do Rio de Janeiro sobre a Produção e 0 Consumo de Transgênicos no Brasil. Revista Eletrônica do Prodema, v. 8, n. 1, p. 84-94, abr. 2014. Disponível em: $<w w w . r e v i s t a r e d e . u f c . b r / r e v i s t a / i n d$ ex.php/rede/article/viewArticle/228 >. Acesso em: 10 maio 2016.

6. Galindo F, Portilho F. "O peixe Morre pela Boca". Como os Consumidores Entendem os Riscos dos Agrotóxicos e dos Transgênicos na Alimentação. Sustentabilidade em Debate, Brasília, DF, v. 6, n. 2, p. 73-87, maio/ago. 2015.

7. Brasil Ministério da Saúde / Conselho Nacional de Saúde. Resolução $n^{\circ} 466$, de 12 de dezembro de 2012. Aprova as seguintes diretrizes e normas regulamentadoras de pesquisas envolvendo seres humanos. Disponível em: <http://www.conselho.saude.gov.br >. Acesso em: 4 nov. 2015.

8. Pedrancini, VD, Corazza-Nunes MJ, Galuch MTB, Moreira ALOR e Ribeiro AC. Ensino e aprendizagem de biologia no ensino médio e a apropriação do saber científico e biotecnológico. Revista Electrônica de Enseñanza de las Ciencias, v. 6, n. 2, p. 299309, 2007. Disponível em: $<$ http://reec.uvigo.es/volumenes/vo lumen6/ART5_Vol6_N2.pdf>. Acesso em: 10 mar. 2016.

9. Calvasina, PG, Silva CMTR, Aguiar GAF, Aguiar MR, Sampaio 
HAC. Conhecimento sobre alimentos geneticamente modificados: um estudo com clientes de um supermercado, situado em área nobre do município de Fortaleza. Revista Brasileira em Promoção da Saúde, Fortaleza, v. 17, n. 1, p. 79-85, 2004.

10. Scare RF, Orati R, Hartung $K$. Conhecimento e comportamento de compra em relação a alimentos transgênicos: um estudo exploratório e comparativo com consumidores brasileiros. In: CONGRESSO DA SOBER, 45., 2007, Londrina. Anais... Londrina: Sociedade Brasileira de Economia, Administração e Sociologia Rural, 2007.
11. Camara MCC, Marinho, CLC, Guilam, MCR, Nodari, RO. Transgênicos: avaliação da possível (in) segurança alimentar através da produção científica. História, Ciências e Saúde, Rio de Janeiro, v. 16, n. 3, p. 669-681, jul./set. 2009.

12. Souza MVF, Hossne WS. Opinião de alunos de graduação em nutrição sobre alimentos transgênicos. Revista BioethiKos, São Paulo, v. 4, n. 4, p. 412-422, 2010.

13. Arnaiz MG. Pensando sobre el riesgo alimentario y su aceptabilidad: el caso de los alimentos transgénicos. Revista de Nutrição, Campinas, v. 17, n. 2, p. 125-49, 2004. 